\title{
High performance and low complexity decoding light-weight video coding with motion estimation and mode decision at decoder
}

\author{
Ted Chih-Wei Lei ${ }^{1 *}$ and Fan-Shuo Tseng ${ }^{2}$
}

\begin{abstract}
Light-weight video coding (LVC) follows distributed video coding (DVC) and designs to move computational complexity from the encoder to the decoder, thus making a low computational complexity encoder. In traditional video coding, the high computational complexity encoder algorithms, where motion estimation and mode decision, are the main transferred objects. In order to alleviate the computational burden, the proposed architecture adopts the Partial Boundary Matching Algorithm (PBMA) and four flexible types of mode decision at the decoder; this circumvents the traditional use of motion estimation and mode decision at the encoder. In simulation, the proposed architecture, Padding Block-based LVC, not only outperforms the state-of-the-art DVC (DISCOVER) codec by up to $4 \sim 5 \mathrm{~dB}$ but also significantly decreases decoder complexity to approximately one hundred times lower than that of the DISCOVER codec.
\end{abstract}

Keywords: Light-weight video coding, Distributed video coding, Padding block-based light-weight video coding, Motion estimation at decoder, Mode decision at decoder

\section{Introduction}

Video coding involves a complementary pair of systems: a compressor (encoder) and a decompressor (decoder). The coding can then be devised to remove any redundancy in the temporal and spatial domains. Generally, two video coding types are typically considered: lossless and lossy coding. Lossy video coding involves motion compensation, transform and quantization processing, while lossless video coding entails entropy coding. In addition, lossy video coding is required for higher compression, since lossless video information only allows for moderate compression. The current standard compatible with the above algorithms has been developed to provide a high quality, low distortion, and low bit-rate transmission. Compared with early proposed standards, the H.264/ AVC standard achieves up to a 50\% improvement in bitrate efficiency and is suitable for many applications, such

\footnotetext{
*Correspondence: tedlei@nkfust.edu.tw

${ }^{1}$ The Electrical Engineering Department of National Sun Yat-sen University,

70 Lienhai Rd., Kaohsiung 80424, Taiwan, Republic of China

Full list of author information is available at the end of the article
}

as web video downloads, video broadcasting, video on demand systems, and consumer electronics video products. However, the above applications of H.264/AVC video coding are subject to numerous complicated loading problems. For example, the video stream is only compressed once, but decoded many times. Typically, the encoder is five to ten times more complex than the decoder. Thus, in order to reduce computational loading at the encoder, the spirit of Distributed Video Coding (DVC) is developed to implement a lower complexity level of video coding, shifting the complexity from the encoder to the decoder without reducing the video coding quality.

The fundamental concept of DVC is based on two significant information theorems: Slepian-Wolf (SW) [1] and Wyner-Ziv (WZ) [2]. The SW coding theorem is a lossless source coding, while the WZ coding theorem is a form of lossy source coding. DVC as defined in [3] must obey these two information theorems; however, the Lightweight Video Coding (LVC) does not follow the original DVC definition. LVC is only complies with the DVC spirit, and develops to implement a lower complexity level of 
video encoding, shifting the complexity from the encoder to the decoder. Based on these theorems, there exist three major groups for the development of DVC architectures: The Stanford DVC scheme [3], the Europe DISCOVER (DIStributed COding for Video sERvices) codec $[4,5]$ and the Berkeley PRISM (Powerefficient, Robust, hIgh-compression, Syndrome-based Multimedia coding) paradigm [6]. Basically, the DVC scheme proposed by Stanford works at the frame level and adopts turbo code-based SW coding; this is characterized by a feedback channel performing rate control at the decoder. The DISCOVER video codec by Europe is actually an extension of the Stanford DVC scheme, which is able to significantly improve performance. The main concept of DISCOVER is to flexibly adjust the GOP-sized selection, and it adopts a low-density parity check accumulator (LDPCA) coding scheme with an 8 b Cyclic Redundancy Checksum (CRC) at the encoder. At the decoder, a bi-directional motion estimation is conducted with spatial smoothing (BiMESS) in order to obtain high quality side information (SI), while motion search is adopted to increase the sub-pixel precision method for BiMESS. The PRISM codec by Berkeley is conducted at the block level and uses the syndrome code-based SW coding. It is characterized by an encoder side rate controller based on the availability of a reference frame.

In addition, Taiwan University has proposed a hybrid DVC (hybrid distributed video coding with frame level coding mode selection) architecture $[7,8]$, which is an extension of the state-of-the-art DVC (DISCOVER) codec. This architecture is beneficial, adding minor computational complexity, and integrating entropy coding into WZ frame encoding, while conventional DVC only uses the channel coding function. Thus, the inclusion of entropy coding not only slightly increases complexity but also improves performance.

Currently, DVC has not reached the performance level of classical inter-frame coding. This is in part due to the quality of the side information (SI), which has a strong impact on the final rate-distortion (RD) performance. In order to produce the SI, DISCOVER uses the MotionCompensated Temporal Interpolation (MCTI) [9] technique. In [10-12], the authors presented DVC schemes that perform the motion estimation both at the encoder and decoder. In [10], the authors propose a pixeldomain DVC scheme, which consists of combining low complexity bit plane motion estimation at the encoder, with motion-compensated frame interpolation at the decoder. The improvements are shown for sequences containing fast and complex motion. In [11], a DVC scheme is presented in which the task of motion estimation is performed both at the encoder and decoder. The results have shown that the cooperation of the encoder and decoder can reduce the overall computational complexity while improving coding efficiency. Finally, [12] proposed combining the global and local motion estimations at the encoder while the motion estimation and compensation are performed both at the encoder and decoder.

Conversely, in [13], the local motion estimation is only performed at the decoder, while the global motion parameters are estimated at the encoder using a scale-invariant feature transform (SIFT) [14] algorithm. It is important to note that the encoding complexity is kept low. The global parameters are sent to the decoder to estimate the global motion compensation (GMC) SI, and the combination between the GMC SI and MCTI SI is made at the decoder. This approach consists of combining global and local motion compensation at the decoder. The parameters of the global motion are estimated at the encoder using SIFT features. These estimated parameters are then sent to the decoder in order to generate a globally motion-compensated SI. Conversely, a locally motion compensated SI is generated at the decoder based on the MCTI of neighboring reference frames. Moreover, an improved fusion of global and local SI during the decoding process is achieved using the partially decoded WZ frames and decoded reference frames. The method proposed in [13] significantly improves the quality of the SI, especially for sequences containing high global motion.

Another DVC paradigm is different to the extension of Stanford DVC scheme and DISCOVER codec. In [15], a dynamic skip mode threshold is proposed, based on PRISM [6] architecture for higher coding efficiency. In the encoder of the classifier module, while the skip mode threshold is a dynamic value different from the original PRISM with fixed value. In the encoder of the syndrome encoding module, the original PRISM architecture, block coefficients in the least significant part were coded in a 4-tuple symbol \{Last, Run, Depth, Path\}, while a 3-tuple symbol \{Last, Run, Path\} was applied in [15] with depth substituted by class type. The key parts of the decoder are the motion search loop, syndrome decoding, and hash checking. First, the motion search is performed at the decoder in order to find suitable predictors. Also, the syndrome decoding module generates side information candidates by searching through previously decoded frames. In addition, the hash checking module checks the correctness of decoded blocks, and the process is repeated until the decoded block passes hash checking, indicating successful decoding.

In [16], on the basis of the original PRISM DVC architecture, a low-complexity feedback channel free DVC architecture is proposed with a new enhance classifier to improve the coding performance. [16] is based on [15] and PRISM architecture, targeting simple video sensors in sensor network applications. An enhanced classifier is proposed at the encoder, which is composed of a light 
motion search module integrated with the classifier for a more accurate rate control, and [15] outperforms other feedback channel free architectures with only a slight increase in encoder complexity. In a feedback-channelfree DVC architecture, the encoder plays a crucial role for coding performance since the bitrate and the quality are determined at the encoder while the decoder is responsible for regular decoding procedures. In light of this, [16] proposes an enhanced classifier architecture in the encoder to further improve the coding performance. However, the [15] can attain the class type and depth distribution of transform coefficients. Also, [16] applies three-step search (3SS) at the encoder of the classifier module, and the 3SS estimates the correlation noise between the current block and the best predictor at the decoder more precisely. The new classifier module exploits the available predictor at the encoder and performs classification to achieve a more accurate rate control. The classifier for class type and depth distribution of transform coefficients is retrained offline.

In fact, DVC avoids the computationally intensive temporal prediction loop at the encoder, by shifting the exploitation of the temporal redundancy to the decoder. This is a significant advantage in a wide range of emerging application scenarios, including wireless video cameras, wireless low-power surveillance, video conferencing with mobile devices, disposable camera, high pollution medical cameras, and visual sensor networks.

DVC effectively reduces the complexity of the encoder, but it also causes some problems. The two main disadvantages are poor performance and high decoder complexity. The cause of poor DVC performance is the DVC encoder's use of only H.264/AVC intra frame coding. This computational complexity is 5 to 10 times lower than that of the traditional H.264/AVC inter frame coding. In addition, conventional DVC has only half the complexity of H.264/AVC intra frame coding between key and WZ frames. Therefore, the usage complexity of the DVC encoder is almost 10 to 20 times lower than the conventional H.264/AVC video coding. It is therefore difficult to achieve the same performance with current traditional video coding. To date, DVC rate distortion (RD) performance remains between H.264/AVC intra and inter frame with no motion video coding. In order to solve this difficult problem, the general solution is the addition of some efficient algorithm in the DVC encoder to improve performance. However, this will mean that the encoder will no longer operate with the original intra frame video coding, but rather with partial inter frame video coding. This will, of course, increase DVC encoder complexity. Another significant problem faced by DVC is high decoding complexity; this problem leads to difficulties in performing real-time video processing. This is the result of the timeconsuming nature of the error-correcting coding of recursive systematic convolutional (RSC) decoding for LDPCA decoders. In [5], it is shown that over $90 \%$ of the computational complexity at the LDPCA decoder is made up of decoding time. Other challenges also remain to be solved in traditional DVC, such as the feedback channel problem. It is clear then that DVC encoder complexity must be reduced, the rate control should be transferred to the decoder, and it must be ensured that that the bidirectional communication channel is available. Many studies have therefore attempted to move the rate control from the decoder back to the encoder and to eliminate the feedback channel problem. Moreover, the encoder requires a larger frame buffer, and in flexible GOP size, this may result in increased hardware costs, especially in slow motion video sequences. The decoder may also encounter the block effect due to the fact that some DVC encoder designs use block-based video coding. Chrominance has no significant effect, and only luminance is considered for processing. Finally, there is no unified DVC standard, which increases the difficulty of making a DVC extension. In summary, then, DVC cannot be widely adopted in many applications due to its poor performance, decoder complexity, and the abovementioned shortcomings.

The proposed LVC scheme, Padding Block-based Light-weight Video Coding (PB-based LVC), solves the two main DVC drawbacks: poor performance and high decoder complexity. Since motion estimation takes up a large part of the computational loading in traditional video coding, the propose method uses a Partial Boundary Matching Algorithm (PBMA) as the motion estimation at the decoder to replace the motion estimation at the encoder. Also another high complexity algorithm, mode decision, needs transfer from the encoder to the decoder through four different flexible modes. This shifting of mode decision to the decoder is a novel move. The proposed scheme therefore differs from the above types of traditional DVC architectures and does not use the traditional DVC frame level design and error correction coding (e.g., Turbo, BCH, or LDPC coding) as the WZ frame coding scheme, but rather uses a block level design and padding-based algorithm.

The remainder of this paper is organized as follows. Section 2 explains DVC in network systems. Section 3 introduces the background (e.g., the traditional motion estimation and mode decision) and methodology (e.g., the PBMA and mode decision at the decoder). Section 4 describes the proposed PB-based LVC architecture. Section 5 discusses the experimental results. Section 6 draws conclusions.

\section{DVC in a network configuration}

The primary concept of DVC is to shift complexity from the encoder to the decoder. However, if it were to be left at that, possible applications would be limited and DVC 
would only be useful in a small number of fields such as wireless video surveillance systems. If, however, DVC were to become applicable to networks, then its potential applications would be very widespread. The description of a traditional video network system and a DVC network system are both as follows.

A traditional video transmission network is a storeand-forward network, where video data flow packets are forwarded hop-by-hop. The content of the video coding data flow is not essentially modified in the network term and transferred directly from the source end to the terminal end. Therefore, in traditional network architecture, the implementation of video encoding and decoding must take place on the terminal equipment without any additional processing in the network term. Thus, the computational loading of all video coding must be completed at the terminal devices. These results are in the high cost of wireless video surveillance, or the need for expensive video compression encoder chips (e.g., ITU-T H.26x and ISO/IEC MPEG-x) $[17,18]$ on commercial mobile phones with camera functions.

In order to effectively reduce the cost of a video transmission network at the encoder, early proposed DVC [3-8] schemes suggested a network solution. In these schemes, the primary aim is to transfer computational complexity from the terminal device to the network term. The advantage of this architecture is that it inherently transfers the complexity to the network term because it uses the DVC scheme in the uplink and a traditional video coding scheme in the downlink. In this network architecture, the computational loading of the terminal device can be reduced as compared to traditional video networks, as shown in Fig. 1.

\section{Related works and main technologies}

In the early stages of DVC development, the video coding standard was based on H.263+, which aimed to develop the motion estimation algorithm. The high performance motion estimation algorithm has a high complexity encoding, which can be considered as the transfer target for DVC. Today, more advanced video coding standards have been proposed. Another outstanding algorithm, mode decision, which flexibly encodes picture blocks in exchange for improving the encoding efficiency, is able to increase the encoding complexity. Mode decision is thus another transfer target. For this reason, the proposed PB-based LVC mitigates the encoder complexity with motion estimation and mode decision. The main idea is that the proposed scheme only adopts zero motion searching, and uses less inter frame encoding. Because motion estimation is not used, a low complexity encoder is expected. At the decoder, the proposed scheme utilizes PBMA and mode decision with decoder algorithms with high performance and low computational complexity inter frame decoding as compared to traditional DVC schemes.

This section contains two subsections: motion estimation [17] and mode decision [18]. Traditional motion estimation at the encoder is discussed in Subsection 3.1, and the proposed primary function of motion estimation at the decoder, PBMA, is introduced in Subsection 3.2. Conventional mode decision at the encoder is explained in Subsection 3.3. Finally, the proposed enhanced function mode decision process at the decoder is demonstrated in Subsection 3.4.

\subsection{Traditional motion estimation at encoder in H.264/ AVC video coding}

All conventional video coding standards use block type motion estimation coding, which is a kind of inter frame motion compensation prediction used for reducing temporal redundancy. The conventional motion estimation operation uses the block of a current frame to search for a best predictor block (best match block) in the search range of the reference frame, where the motion vector represents the best match block position with a zero motion block. The rate distortion optimization (RDO) function-a general assessment method used to achieve the best mode between

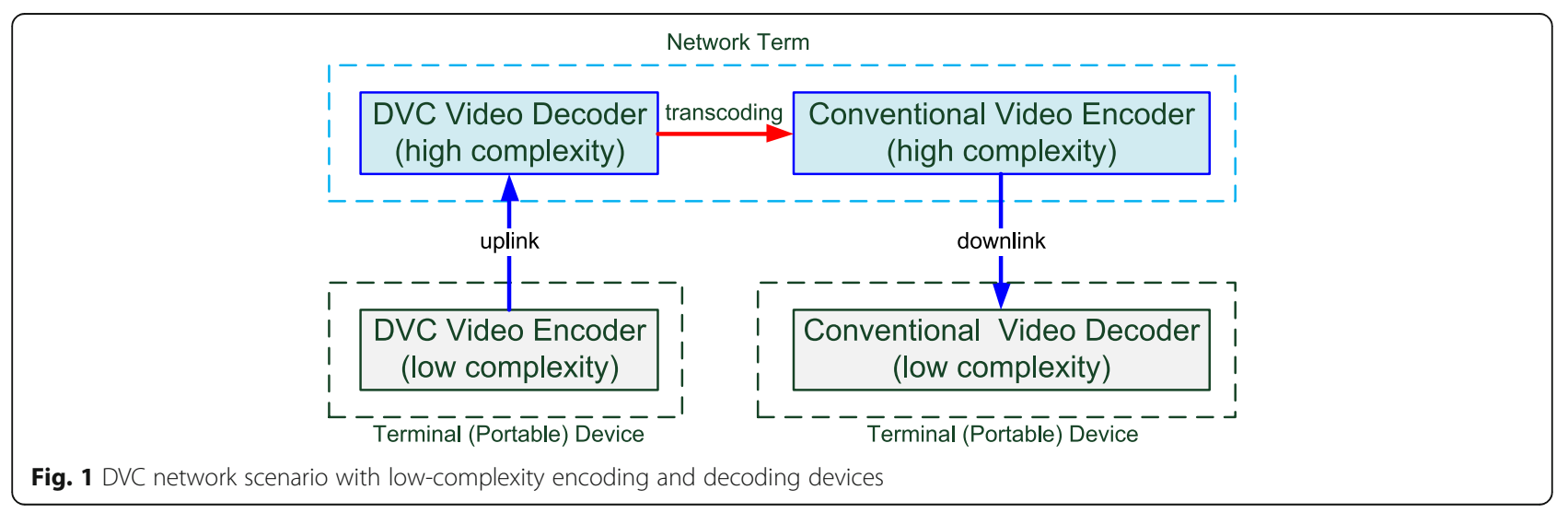


performance and rate of data flow-of the motion estimation can be expressed as follows:

$$
\begin{aligned}
\mathrm{J}_{\mathrm{ME}}\left(\overrightarrow{\mathrm{m}}, \mathrm{F}_{\text {ref }} \mid \lambda_{\mathrm{ME}}\right)= & \operatorname{SAD}\left(\mathrm{s}, \mathrm{c}\left(\mathrm{F}_{\text {ref }}, \overrightarrow{\mathrm{m}}\right)\right) \\
& +\lambda_{\mathrm{ME}} \cdot\left(\mathrm{R}(\overrightarrow{\mathrm{m}}-\overrightarrow{\mathrm{p}})+\mathrm{R}\left(\mathrm{F}_{\text {ref }}\right)\right)
\end{aligned}
$$

where ME denotes motion estimation, $\overrightarrow{\mathrm{m}}=\left(\mathrm{m}_{\mathrm{x}}, \mathrm{m}_{\mathrm{y}}\right)^{\mathrm{T}}$ $=(\mathrm{dx}, \mathrm{dy})^{\mathrm{T}}$ is the motion vector $(\mathrm{T}$ is a transpose matrix), $F_{\text {ref }}$ is the reference frame, and $\lambda_{\mathrm{ME}}$ is the motion estimation Lagrange multiplier. The SAD function is the Sum of Absolute Differences, while s and c are the original reference video signals. $R(\vec{m}-\vec{p})$ represents the bit coding for the motion vector, and $R\left(F_{\text {ref }}\right)$ is the bit coding for the reference frame. In motion estimation, a motion vector is selected by the SAD, and the SAD is computed as:

$$
\operatorname{SAD}\left(\mathrm{s}, \mathrm{c}\left(\mathrm{F}_{\mathrm{ref}}, \overrightarrow{\mathrm{m}}\right)\right)=\sum_{\mathrm{x}=1, \mathrm{y}=1}^{\mathrm{N}, \mathrm{s}}|\mathrm{s}(\mathrm{x}, \mathrm{y})-\mathrm{c}(\mathrm{x}-\mathrm{dx}, \mathrm{y}-\mathrm{dy})|
$$

where $\mathrm{s}$ and $\mathrm{c}$ are the original reference video signal, $\mathrm{F}_{\text {ref }}$ is the reference frame, $\overrightarrow{\mathrm{m}}$ is the motion vector, $\mathrm{N}$ is block size, $(x, y)$ is a pixel of the reference frame, and $(\mathrm{d} x, \mathrm{dy})$ indicates the motion vector. However, encoder complexity is caused by the accumulative additions involved in motion search. Therefore, Eq. (2) shows that the encoder incurs high complication encoder loading with motion estimation, as depicted in Fig. 2.

\subsection{Motion estimation at decoder with PBMA}

The primary aim of LVC is to transfer certain complicated operations from the encoder to the decoder. During the transfer, the decoding performance will not be severely degraded and will remain within a tolerable range. The high performance motion estimation at the encoder of the transitional video coding is essentially a high computational complexity algorithm. However, the proposed scheme does not utilize motion estimation at the encoder. This significantly reduces the encoding complexity, and without high efficiency motion estimation, the performance may be degraded outside the predefined tolerable range. The proposed scheme uses PBMA to replace the function of motion estimation, and PBMA can thus demonstrate performance that approaches motion estimation at the encoder. The proposed PBMA algorithm is detailed as follows:

$$
\begin{aligned}
& \operatorname{PBMup}_{\text {region }}(\mathrm{dx}, \mathrm{dy})=\sum_{\mathrm{X}=\mathrm{X}_{0}-\mathrm{b}}^{\mathrm{X}_{0}+\mathrm{N}-1} \mid \mathrm{P}_{\text {curr }}\left(\mathrm{X}, \mathrm{Y}_{0}-\mathrm{b}\right) \\
& +\sum_{\mathrm{X}=\mathrm{X}_{0}-\mathrm{b}}^{\mathrm{X}_{0}+\mathrm{N}-1} \mid \mathrm{P}_{\text {curr }}\left(\mathrm{X}, \mathrm{Y}_{0}-\mathrm{b}+1\right) \\
& +\sum_{X=X_{0}-b}^{X_{0}+N-1} \mid P_{\text {curr }}\left(X, Y_{0}-b+2\right) \\
& -\operatorname{Pref}\left(\mathrm{X}+\mathrm{dx}, \mathrm{Y}_{0}-\mathrm{b}+2+\mathrm{dy}\right) \\
& +\sum_{X=X_{0}-b}^{X_{0}+N-1} \mid P_{\text {curr }}\left(X, Y_{0}-1\right) \\
& -\operatorname{Pref}\left(\mathrm{X}+\mathrm{dx}, \mathrm{Y}_{0}-1+\mathrm{dy}\right)
\end{aligned}
$$

, and

$$
\begin{aligned}
& \text { PBMleft }_{\text {region }}(\mathrm{dx}, \mathrm{dy})=\sum_{\mathrm{Y}=\mathrm{Y}_{0}}^{\mathrm{Y}_{0}+\mathrm{N}-1} \mid \mathrm{P}_{\text {curr }}\left(\mathrm{X}_{0}-\mathrm{b}, \mathrm{Y}\right) \\
& +\sum_{\mathrm{Y}=\mathrm{Y}_{0}}^{\mathrm{Y}_{0}+\mathrm{N}-1} \mid \mathrm{P}_{\text {curr }}\left(\mathrm{X}_{0}-\mathrm{b}+1, \mathrm{Y}\right) \\
& +\sum_{\mathrm{Y}=\mathrm{Y}_{0}}^{\mathrm{Y}_{0}+\mathrm{N}-1} \mid \mathrm{P}_{\text {curr }}\left(\mathrm{X}_{0}-\mathrm{b}+2, \mathrm{Y}\right) \\
& +\sum_{\mathrm{Y}=\mathrm{Y}_{0}}^{\mathrm{Y}_{0}+\mathrm{N}-1} \mid \mathrm{P}_{\text {curr }}\left(\mathrm{X}_{0}-1, \mathrm{Y}\right) \\
& -\operatorname{Pref}\left(X_{0}-b+2+d x, Y+d y\right) \mid \\
& -\operatorname{Pref}\left(X_{0}-1+d x, Y+d y\right)
\end{aligned}
$$

, then

$$
\begin{aligned}
\operatorname{PBM}(\mathrm{dx}, \mathrm{dy})= & \mathrm{PBM}_{\mathrm{up}_{\text {region }}}(\mathrm{dx}, \mathrm{dy}) \\
& +\mathrm{PBM}_{\text {left }_{\text {region }}}(\mathrm{dx}, \mathrm{dy})
\end{aligned}
$$

where $\mathrm{PBM}(\mathrm{dx}, \mathrm{dy}), \mathrm{PBM}_{\text {up_region }}(\mathrm{dx}, \mathrm{dy})$, and $\mathrm{PBM}$ _left_region

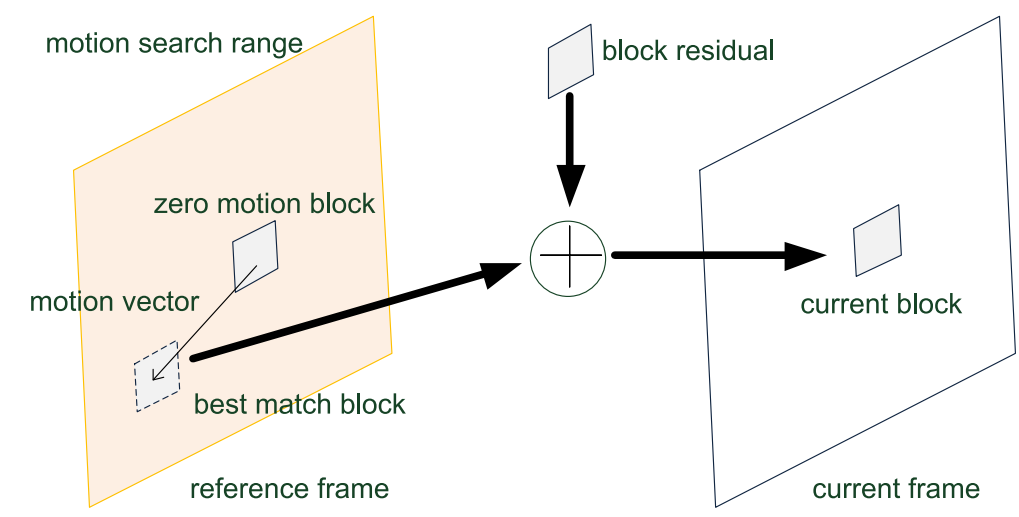

Fig. 2 Motion estimation at the encoder in traditional video coding 
(dx,dy) are the total, upper region, and left side SAD of PBMA and $(\mathrm{dx}, \mathrm{dy})$ is a candidate motion vector. $\mathrm{P}_{\text {curr }}(\mathrm{X}, \mathrm{Y})$ and $\mathrm{P}_{\text {ref }}(\mathrm{X}, \mathrm{Y})$ denote the pixel value of current and reference frame. $\left(\mathrm{X}_{0}, \mathrm{Y}_{0}\right)$ is the position of the skipped block. Here, $\mathrm{N}$ is block size and $\mathrm{b}$ is the condition size of the template neighbor region.

PBMA is modified from the Boundary Matching Algorithm (BMA) [19]. BMA is a kind of error concealment method and mainly uses the boundary pixels of loose blocks to find the best matching block in the search range of a reference frame. The steps involved in BMA are as follows: First, the BMA template is the neighborhood pixel of the loose block. Second, as in motion estimation, the candidate block is selected from the search range in the reference frame. Third, in the search range, each candidate block neighborhood pixel is compared with the template. Fourth, the candidate block, which is most similar to the template, is the best matching block. Finally, the best matching block is pasted back into the current frame. The major difference between PBMA and BMA is that BMA uses all adjacent pixels of the block as a template, whereas PBMA only uses partial adjacent pixels (in general, only two adjacent pixels) because the decoding block of adjacent pixels has not been decoded. Thus, only the partial block adjacent pixels can be used. As shown in Fig. 3, each small block (white and light blue colors) represents one pixel.

\subsection{Conventional mode decision at encoder in H.264/AVC} video coding

Conventional video coding can improve block selection flexibility as well as the ratio of block comparison error in the mode decision algorithm; eight different block mode selections are considered, e.g., $16 \times 16,16 \times 8,8 \times$ $16,8 \times 8,8 \times 4,4 \times 8$, and $4 \times 4$. The use of more block selections with differing flexibility macroblock types regarding the motion estimation and motion compensation will significantly enhance performance but will also increase the computational complexity at the encoder. In addition, the mode decision according to the frame complex generally uses different block modes, where the complex part uses smaller macroblocks, and the smooth part represents larger macroblocks, as shown in Fig. 4.

The RDO function of mode decision is defined as follows:

$$
\mathrm{J}_{\mathrm{MD}}\left(\mathrm{s}, \mathrm{c}, \mathrm{MD} \mid \lambda_{\mathrm{MD}}\right)=\operatorname{SSD}(\mathrm{s}, \mathrm{c}, \mathrm{MD})+\lambda_{\mathrm{MD}} \cdot \mathrm{R}(\mathrm{s}, \mathrm{c}, \mathrm{MD})
$$

where $\mathrm{s}$ and $\mathrm{c}$ are the original reference video signals, MD denotes mode decision, and $\lambda_{\mathrm{MD}}$ is the mode decision Lagrange multiplier. SSD is the Sum of Square Difference between the original frame and the reference frame. $\mathrm{R}(\mathrm{s}, \mathrm{c}, \mathrm{MD})$ represents the bit coding between the original frame and the reference frame. In mode decision, the luminance SSD is defined as:

$$
\operatorname{SSD}(\mathrm{s}, \mathrm{c}, \mathrm{MD})=\sum_{\mathrm{x}=1, \mathrm{y}=1}^{\mathrm{N}, \mathrm{N}}|\mathrm{s}(\mathrm{x}, \mathrm{y})-\mathrm{c}(\mathrm{x}, \mathrm{y})|^{2}
$$

where $\mathrm{s}$ and $\mathrm{c}$ are the original reference video signals, MD denotes mode decision, $\mathrm{N}$ is block size, and $(\mathrm{x}, \mathrm{y})$ is a pixel in the reference frame. Similarly, mode selection encounters the tradeoff between performance and

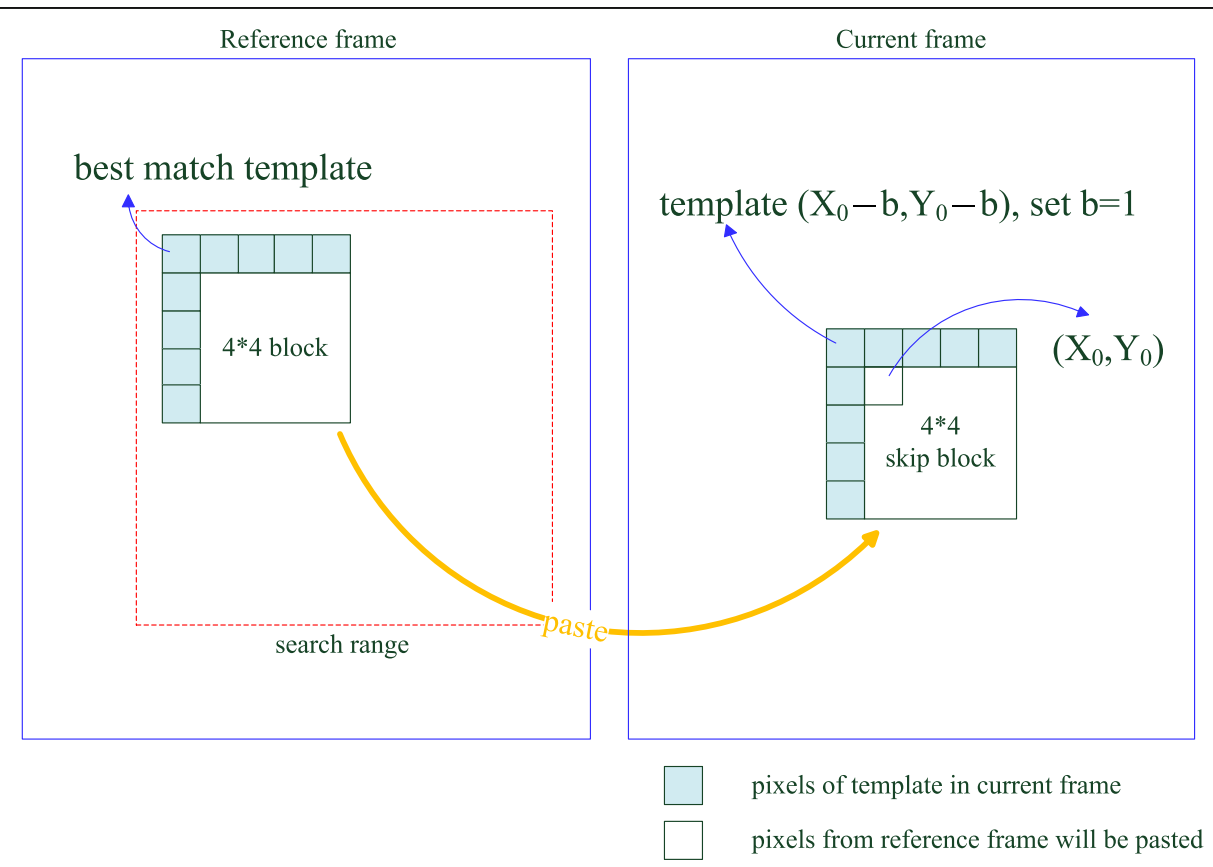

Fig. 3 Matching region of PBMA (motion estimation at the decoder) 

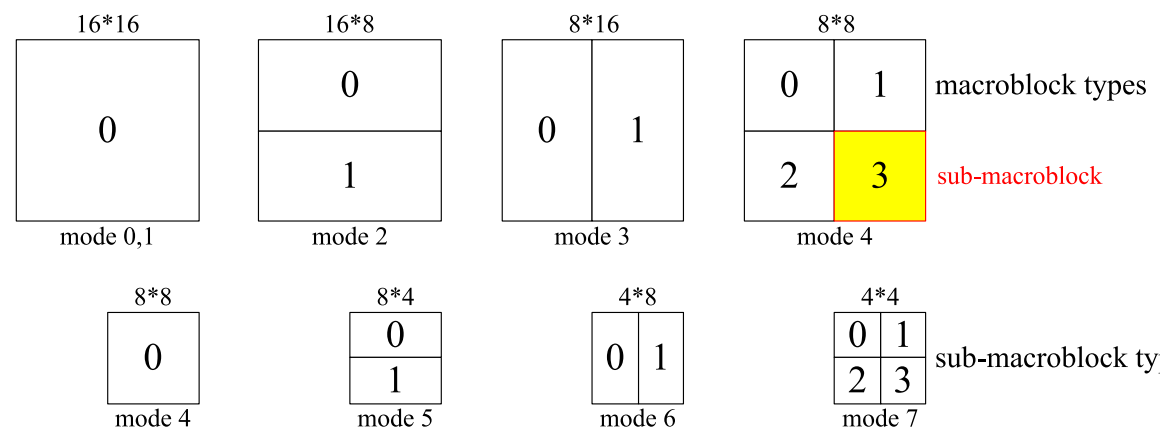

$$
\begin{array}{|l|l|}
\multicolumn{2}{|c}{4 * 4} \\
\hline 0 & 1 \\
\hline 2 & 3 \\
\hline \text { mode } 7
\end{array} \text { sub-macroblock types }
$$

Fig. 4 Mode types in mode decision at encoder with conventional H.264/AVC video coding (the number within each block indicating the coding order)

complexity issues, as with the traditional motion estimation algorithm.

\subsection{Mode decision at decoder with PB-based LVC}

Although mode decision performance can be significantly enhanced in conventional video coding, the computational complexity at the encoder will remain high. Therefore, the proposed scheme aims to shift mode decision to the decoder; the primary goal is to use its high performance characteristics to address the traditional DVC low performance problem. The proposed scheme, with mode decision at the decoder, largely strengthens PBMA which is a motion estimation at the decoder algorithm, and effectively improves performance over using PBMA without mode decision at the decoder. This is the first time mode decision has been shifted to the decoder. In addition, the proposed scheme is a lowcomplexity mode decision at the decoder along with traditional high-complexity decoder DVC solutions. The proposed mode decision at the decoder has four different modes: modes 0 to 3 , where the mode (block type) is chosen from the candidate type set $\{4 \times 4,4 \times 2$, $2 \times 4,2 \times 2\}$. Apart from mode 0 , the other modes may fail to be completed because neighborhood blocks have not been decoded. The selection method of best block types involves calculating the mean addition differential (MAD) by neighborhood pixels. Next, the best type block is pasted back in order, and mode decision at the decoder is completed, as depicted in Fig. 5. Therefore, the MAD is defined as:

$$
\operatorname{MAD}(x, y)=\operatorname{SAD}(x, y) / N^{2}
$$

where $\operatorname{SAD}(x, y)$ is the pixel value of the Sum of Absolute Differences and $\mathrm{N}$ is the block size.

\section{Padding block-based light-weight video coding}

The PB-based LVC architecture is comprised of three parts at the encoder: the classifier, skip block mask and rearrangement (including the skip block record table), and the conventional intra frame encoder, depicted in the Fig. 6. Initially, the classifier can be divided into SAD and DC classifiers. The SAD classifier is used to determine zero motion blocks. After the SAD classifier, the DC (Direct Current) classifier is carried forward to replace the search of low motion blocks for motion estimation (DC value is generally used in the $\mathrm{DC}$ coefficient of DCT (discrete cosine transform)). Therefore, the classifier is only suitable for recognizing zero and low motion blocks and is unsuitable for other cases, especially in high motion blocks. Two functions, the skip block mask and rearrangement, are based on the results of the classifier. If the classification value is smaller than the setting threshold, the block will be skipped, and the DC value of the block will be filled in; otherwise, the block will be retained and sent to the conventional intra frame encoder after further transferring the video stream to the decoder. The skip blocks with DC fill in function have better performance than without DC fill in function. Finally, the skipped blocks' data are saved in the skip block record table with three states (non-skip blocks, SAD classification blocks, and DC classification blocks). The encoded video stream and record table are then output to the decoder, respectively.

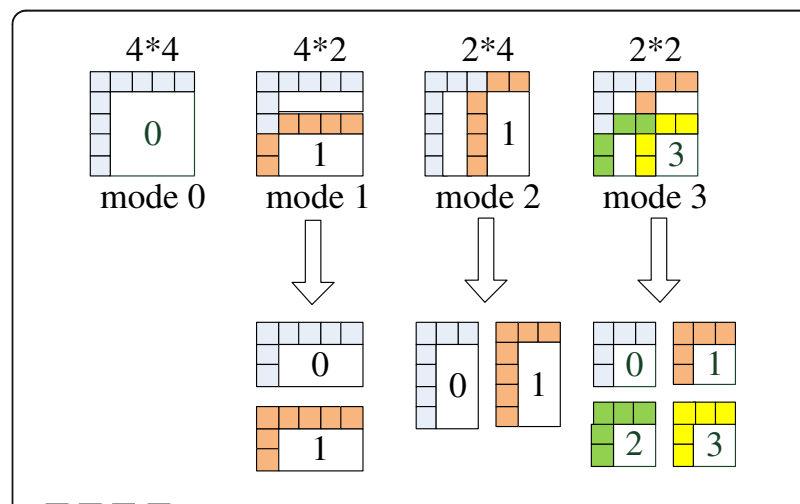

$\square \square \square \square$ pixels of template in candidate block neighborhood

Fig. 5 Mode decision at the decoder with PB-based LVC (the number within each block indicates the coding order) 


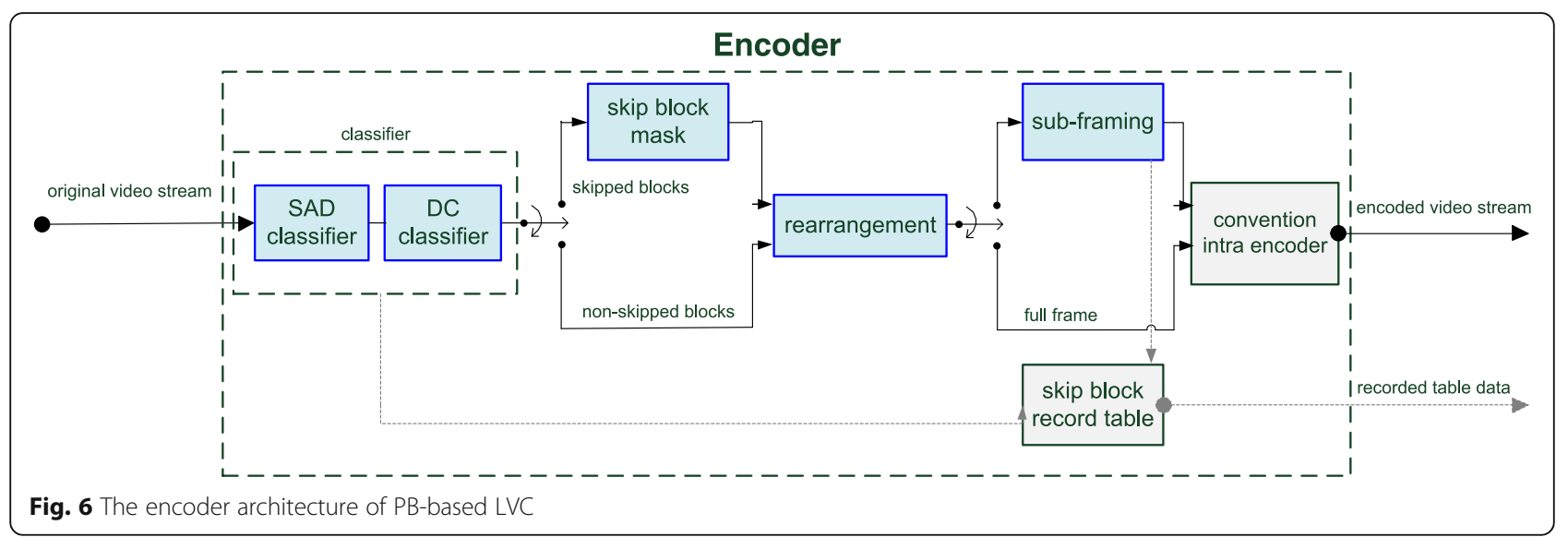

The decoder consists of three main parts, including the conventional intra frame decoder, the block padding and the pixel padding, shown in the Fig. 7. The conventional intra frame decoder first decodes the video stream from the encoder. After decoding, the block padding is used to pad blocks and is divided into Zero Motion Vector Replacement (ZMVR) and Partial Boundary Matching Algorithm (PBMA) to replace the high complexity motion estimation algorithm at the encoder. ZMVR and PBMA acquire the skip block record table information from the skipped blocks of the encoder and then pad zero and low motion blocks with four flexible block mode decision. The PBMA algorithm is employed to select the best matching block by neighbor pixel data around the skipped block and searches candidate blocks as with motion estimation in the setting search range, after finding the best matched block and then padding it from the reference frame. After block padding, the remaining unrecovered blocks use pixel padding. Pixel padding consists of Spatial Temporal Texture
Synthesis (STTS) and Pixel Interpolation (PxI). STTS is an efficient approach not only for image reconstruction technology but also for video compression. Of course, STTS finds the best matched pixel with four neighborhood templates from the appropriate search range. The proposed STTS algorithm not only uses spatial frames as a search range but also uses temporal frames. Finally, the PxI is used to complete the padding for all remaining unrecovered pixels after the above algorithm. The PxI utilizes image inpainting technology to recover pixels.

\subsection{Encoder}

The proposed scheme adopts a GOP frame coding structure, as found in traditional video coding. Herein, the first frame is encoded with the traditional intra frame encoding, and the other frames function using skip block encoding. With this procedure, as with the traditional video coding results, intra frame coding prevents the entire GOP frame from distorting.

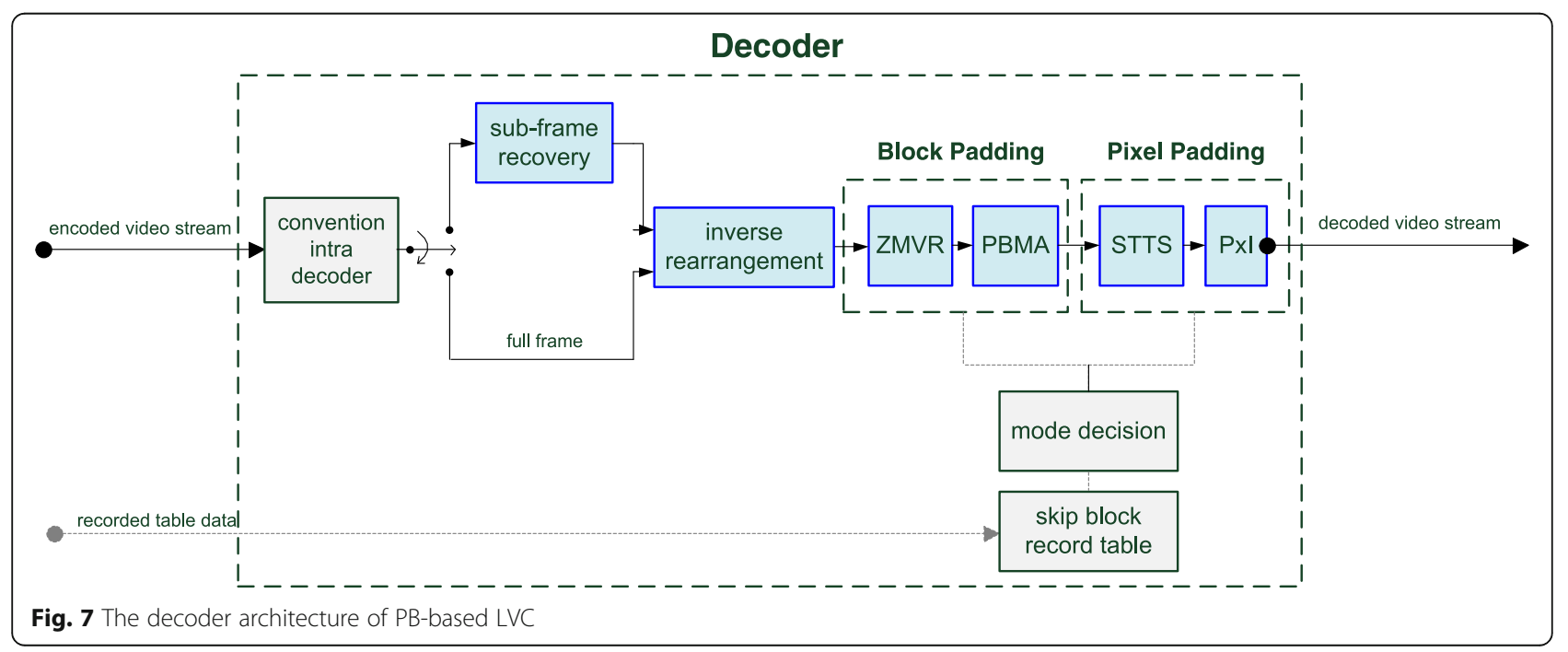




\subsubsection{Classifier}

The functional block, classifier, includes the SAD and DC classifiers. These classifiers are used to identify the zero motion block and the low motion block; therefore, this design is not suitable for determining the medium and high motion blocks. The SAD classifier could be used to determine SAD (0) (the zero motion block). The formula is defined as follows:

$$
\operatorname{SAD}(x, y)=\left|\sum_{x=x 0}^{x 0+N-1} \sum_{y=y 0}^{y 0+N-1} \operatorname{Bcurr}(x, y)-\operatorname{Bref}(x, y)\right|
$$

where $\operatorname{SAD}(x, y)$ is the $\operatorname{SAD}$ value between the current and reference block, $N$ is block size, $(x 0, y 0)$ are the coordinates of the current block, $\mathrm{B}_{\text {curr }}(x, y)$ and $\mathrm{B}_{\text {ref }}(x, y)$ are the pixel value of the current and reference blocks. After the SAD classifier, the DC classifier is performed and the DC value (average value) is evaluated. This is calculated as

$$
\begin{aligned}
\operatorname{AVG}(\mathrm{x}, \mathrm{y}) & =\operatorname{AVG}_{\mathrm{curr}}(x, y)-\mathrm{AVG}_{\mathrm{ref}}(x, y) \\
& =N^{-2} \mid \sum_{x=x 0}^{x 0+N-1} \sum_{y=y 0}^{y 0+N-1} \operatorname{Bcurr}(x, y) \\
& -\sum_{x=x 0}^{x 0+N-1} \sum_{y=y 0}^{y 0+N-1} \operatorname{Bref}(x, y) \mid
\end{aligned}
$$

where $\mathrm{AVG}_{\text {curr }}(x, y)$ and $\mathrm{AVG}_{\text {ref }}(x, y)$ are the $\mathrm{DC}$ values (average value) of the current and reference blocks, respectively; $N$ is the block size, $(x 0, y 0)$ is the pixel position of the current block, $\mathrm{B}_{\text {curr }}(x, y)$ and $\mathrm{B}_{\text {ref }}(x, y)$ are pixel values of the current and reference blocks. This average value helps to easily search for low motion blocks when the low motion block partially overlaps pixels of the co-located block. Therefore, with the classifier-block, it is easy to see that the proposed LVC encoder adopts partial inter frame coding, rather than the pure intra frame coding adopted by traditional DVC.

\subsubsection{Skip block mask and rearrangement}

The functional block, skip block mask, first masks all skip blocks with the results obtained from the classifier-block and saves the information of the skip blocks to the skip block record table. The mask condition is designed as

$$
\mathrm{th}_{\mathrm{sad}}=\mathrm{SAD}(x, y), \mathrm{th}_{\mathrm{dc}}=\operatorname{AVG}(x, y)
$$

$$
\begin{aligned}
\operatorname{mask}_{\mathrm{sad}}(i) & = \begin{cases}1, & \mathrm{th}_{\mathrm{sad}}(i) \leq \mathrm{\tau}_{1} \\
0, & \text { otherwise }\end{cases} \\
\text { mask }_{\mathrm{dc}}(\mathrm{i}) & = \begin{cases}1, & \mathrm{th}_{\mathrm{dc}}(i) \leq \mathrm{\tau}_{2} \\
0, & \text { otherwise }\end{cases}
\end{aligned}
$$

where th ${ }_{\text {sad }}$ and th ${ }_{\mathrm{dc}}$ are the $\operatorname{SAD}(x, y)$ and $\operatorname{AVG}(x, y)$ differential values from Eqs. (9) and (10), respectively. Here, $\operatorname{mask}(i)$ is set to 1 , and if the the and $t_{\mathrm{dc}}$ of block $i$ are below the thresholds $\tau_{1}$ and $\tau_{2}$, the block is skipped. Otherwise, it is assumed to be a non-skip block. After the skip block function, the rearrangement-block function will rearrange reserve (non-skip) blocks by a new order, which concentrates non-skipped blocks together. As a result, it is easy to distinguish skip blocks and non-skip blocks in a frame.

\subsubsection{Sub-framing}

After the rearrangement-block is the sub-framing-block, which is a half video frame processing, if this frame skips over $50 \%$ of the blocks. Otherwise, if the frame skips less than $50 \%$ of the blocks, full video frame is retained. From this, the frame size transmitted to the decoder can be greatly reduced in slow motion video sequences. However, if the frame in the high motion video sequences is insufficiently conditioned, it will send a full frame to the next functional block.

\subsubsection{Conventional intra frame encoder}

The functional block of the conventional intra frame encoder, such as H.263+, H.264/AVC, H.265/HEVC, MPEG-2 and MPEG-4 intra frame coding, and even JPEG, JPEG-2000, all depend on the range of suggestion. Therefore, high-performance H.264/AVC main profile and H.265/HEVE main profile level 1 intra frame video coding is adopted in this paper. In addition, since the rate control issue arises from the feedback channel problem, the proposed scheme includes a rate control at the encoder, which differs fundamentally from conventional DVC with the rate control at the decoder.

\subsubsection{Skip block record table}

As described above, this record table needs only $2 \mathrm{~b}$ to store information per block, e.g., $(0,0)$ stands for non-skip block, $(1,0)$ refers to skip blocks by $\operatorname{SAD}$ classifier, $(0,1)$ is skip blocks by DC classifier, and $(1,1)$ means reserved.

\subsection{Decoder}

The decoder processing kernel is under the skip block record table information. Therefore, this kernel can generate a high-performance decoder with the block and pixel padding functions. The considered decoder contains the following parts. 


\subsubsection{Conventional intra frame decoder}

The proposed scheme uses H.264/AVC and H.265/ HEVC video decoding directly and can be used to detect most of the parameters from the encoder automatically; this excludes skip block information.

\subsubsection{Sub-frame recovery}

If the encoder uses a sub-framing function, the decoder should recover it to a full fame; if not, skip this step.

\subsubsection{Inverse rearrangement}

The functional block, inverse rearrangement, is arranged in order of the blocks. The blocks' positions are recovered to form the original video frame status.

\subsubsection{ZMVR}

The ZMVR is used to directly paste the zero motion blocks from the co-located block of the reference (previous) frame and according to the information from the skip block record table. However, sometimes this is unsatisfactory because some non-skip blocks have not been reconstructed from the reference frame, and the non-reconstructed blocks will thus continue to be processed in the next PBMA-block.

\subsubsection{PBMA}

The PBMA function, which is a boundary matching algorithm, primarily uses the boundary pixels of loose blocks to find the best match blocks in the search range of the reference frame. The steps of PBMA are as follows: First, the PBMA template is the upper and left hand side neighborhood pixel of the lose block. Second, the candidate block is selected from the search range in the reference frame. Third, each candidate block's neighborhood pixel is compared with the template in the search range. Fourth, the candidate block, which is most similar to the template, is the best match block. Finally, the best match block is pasted back into the current frame. For simplicity, the calculation related to PBMA is defined as follows:

$$
\begin{aligned}
\operatorname{PBMup}_{\text {region }}(\mathrm{dx}, \mathrm{dy}) & =\sum_{\substack{X=X_{0}-1 \\
-}}^{X_{0}+N-1} \mid \mathrm{P}_{\text {curr }}\left(X, Y_{0}-1\right) \\
& \operatorname{Pref}\left(X+\mathrm{dx}, Y_{0}-1+\mathrm{dy}\right) \mid
\end{aligned}
$$

and

$$
\begin{aligned}
\text { PBMleft }_{\text {region }}(\mathrm{dx}, \mathrm{dy}) & =\sum_{Y=Y_{0}}^{Y_{0}+N-1} \mid \mathrm{P}_{\text {curr }}\left(X_{0}-1, Y\right) \\
& -\operatorname{Pref}\left(X_{0}-1+\mathrm{dx}, Y+\mathrm{dy}\right) \mid
\end{aligned}
$$

, and finally
$\operatorname{PBM}(d x, d y)=\operatorname{PBM}_{\text {up }_{\text {region }}}(\mathrm{dx}, \mathrm{dy})+\operatorname{PBM}_{\text {left }_{\text {region }}}(\mathrm{dx}, \mathrm{dy})$

where Eqs. (13), (14), and (15) are defined similar to Eqs. (3), (4), and (5) above, except b is set to 1.

\subsubsection{STTS}

The STTS function is a kind of texture synthesis algorithm and differs from the conventional texture synthesis which applies the spatial domain only. However, STTS uses the spatial frame and refers to the temporal frame. Although this will increase complexity, performance is enhanced as well. Spatial texture synthesis $[20,21]$ is one of the more efficient approaches used to reconstruct a large digital image from a small digital sample image in conventional image processing. This is done by utilizing its structural content. Thus, the proposed scheme uses this algorithm to implement the pixel padding.

After the block padding function, most blocks have been recovered, and only a few blocks need to be reconstructed by pixel padding. STTS adopts 8-neighborhoods as a search range at the decoder. Finding the best match of the current pixel involves using the template block on the four sides of each individual current pixel; the template is on the upper, lower, left, and right, respectively. Then, the best match of the template block in the search range is found. Finally, if the candidate pixel is selected, the candidate pixel is pasted to recover it, as depicted in Fig. 8.

\subsubsection{PxI}

The functional block, PxI, utilizes pixel interpolation to reconstruct pixels in the current frame. The PxI-block can use any subjection interpolation algorithm and uses the average value of 4-neighborhoods pixels to complete unrecovered pixels.

\subsubsection{Skip block record table}

The function, skip block record table, is based on the table from the encoder. This table is able to support the best decoding information.

\subsection{Enhance function}

The proposed enhance function includes the following:

\subsubsection{Backward video sequence procedure}

After the block padding is implemented with the forward procedure, the backward video sequence procedure processes the unrecovered blocks again using the PBMA block. This may recover some blocks that could not be recovered in the forward procedure. This method is able to recover more blocks and promotes better performance. 


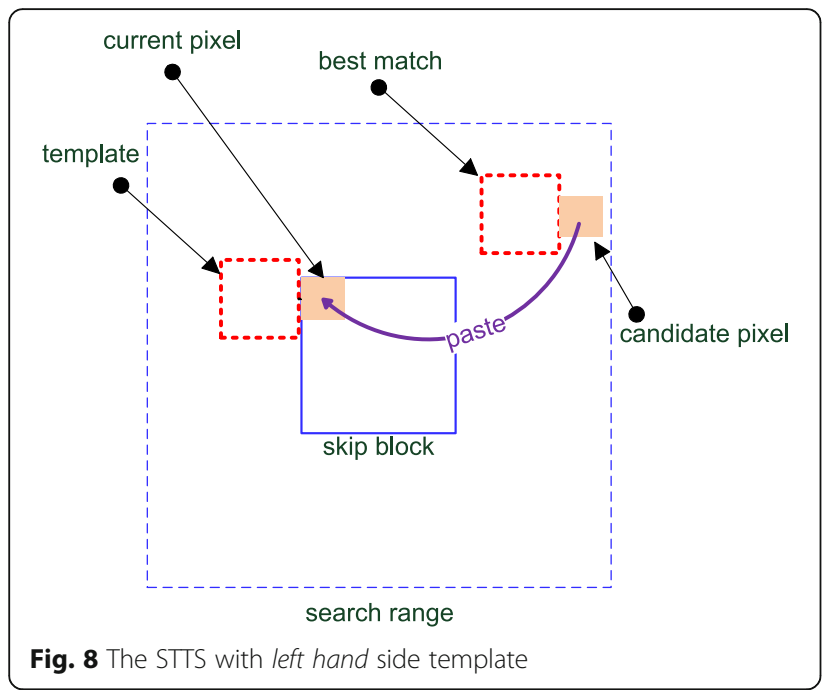

Fig. 8 The STTS with left hand side template

\subsubsection{Mode decision at decoder}

As mentioned above, mode decision at the decoder primarily enhances the PBMA function in block padding, because the PBMA only processes the $\{4 \times 4\}$ blocks. Mode decision at the decoder then increases different block sizes for template matching. The calculation is the same as in conventional video coding and uses MAD to distinguish different block sizes for different modes. The departure from traditional video coding is that the MAD value cannot be calculated for each mode, where the minimum value is selected as the best mode. It is made in order of the candidate set $\{4 \times 4,4 \times 2,2 \times 4,2 \times 2\}$. If decoding cannot be performed for the last mode block, the next mode cannot be decoded. Thus, only decoded modes can be used for a comparison, and the minimum MAD value is selected as the best block.

\subsubsection{Remaining available enhancement functions}

There are still more available enhancement functions which could be used in the PB-based LVC, e.g., mutual
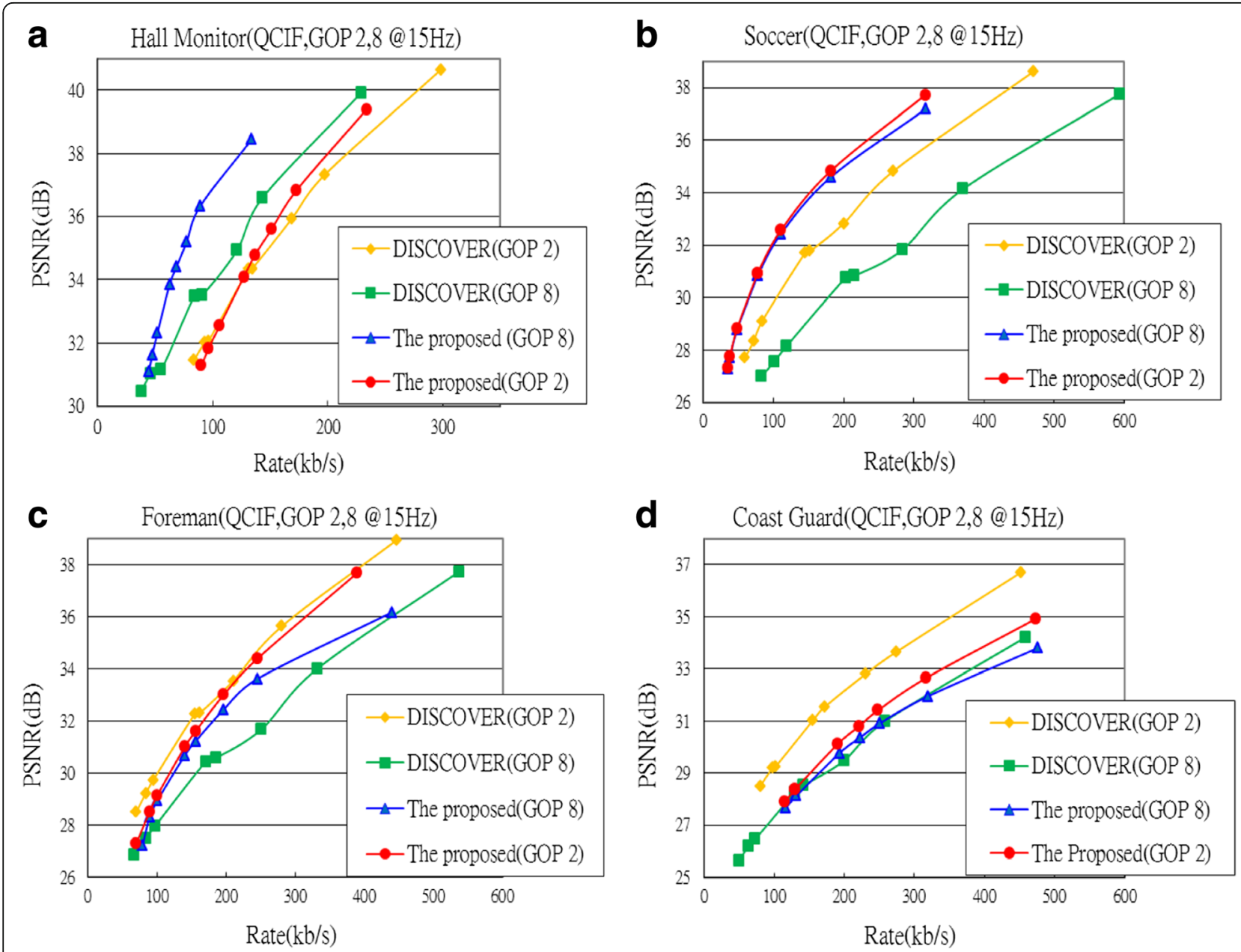

Fig. 9 The RD performance comparison of the proposed PB-based LVC and the DISCOVER codec 
bi-directional frame coding at the decoder, multiple reference frame coding at the decoder, sub-pixel motion search at the decoder for PBMA , and a de-blocking filter for block based LVC. The above subjection enhancement functions can be applied as effective methods for improving PB-based LVC.

\section{Experiment results}

In the experiments, the RD performance and computation time of the proposed LVC parameters are compared with those of the state-of-the-art DISCOVER codec, which is a typical DVC architecture, and most of the literature entries selected are compared with it.

The RD performance of the proposed scheme is compared with that of the DISCOVER codec; therefore, all parameters follow DISCOVER, e.g., JM reference software, and the main profile is used to compare the PSNR of luminance $(\mathrm{Y})$ without comparison with chrominance $(\mathrm{U}$, $\mathrm{V})$ levels. It is assumed that the channel is free, frame rate is $15 \mathrm{~Hz}$, and quantization parameters QPs and QI are identical with those of DISCOVER. GOP length is 2 and 8 , and the total number of frames is 150 . Four general video test sequences, "Hall Monitor," "Foreman," "Soccer," and
"Coast Guard," are selected, where the Hall Monitor, Foreman, and Soccer video test sequences are low, medium, and high activity video test sequences, respectively. Notably, the Coast Guard sequence is a significant one.

In the context of computational complexity, in order to avoid the difference in execution time for different platforms (CPU time), the ratio against H.264/AVC intra frame coding is used instead of a comparison of execution time directly. Here, the personal computers (PC) associated with Intel Pentium dual-core CPU processor at $1.3 \mathrm{GHz}$ and $4 \mathrm{~GB}$ RAM at $1.3 \mathrm{GHz}$ are installed with the Microsoft Windows 7 64-b operating system.

\subsection{RD performance}

Overall, the proposed scheme's performance (red and blue line) is better than that of DISCOVER (orange and green line) in most video test sequences, except Coast Guard as it is a significant video test sequence and very suited for the DISCOVER codec; thus, surpassing its ability is not easily accomplished. In GOP 8 , the proposed scheme's performance (blue line) is better than that of DISCOVER. This is different in Coast Guard as the proposed scheme exhibits a loss of performance. But
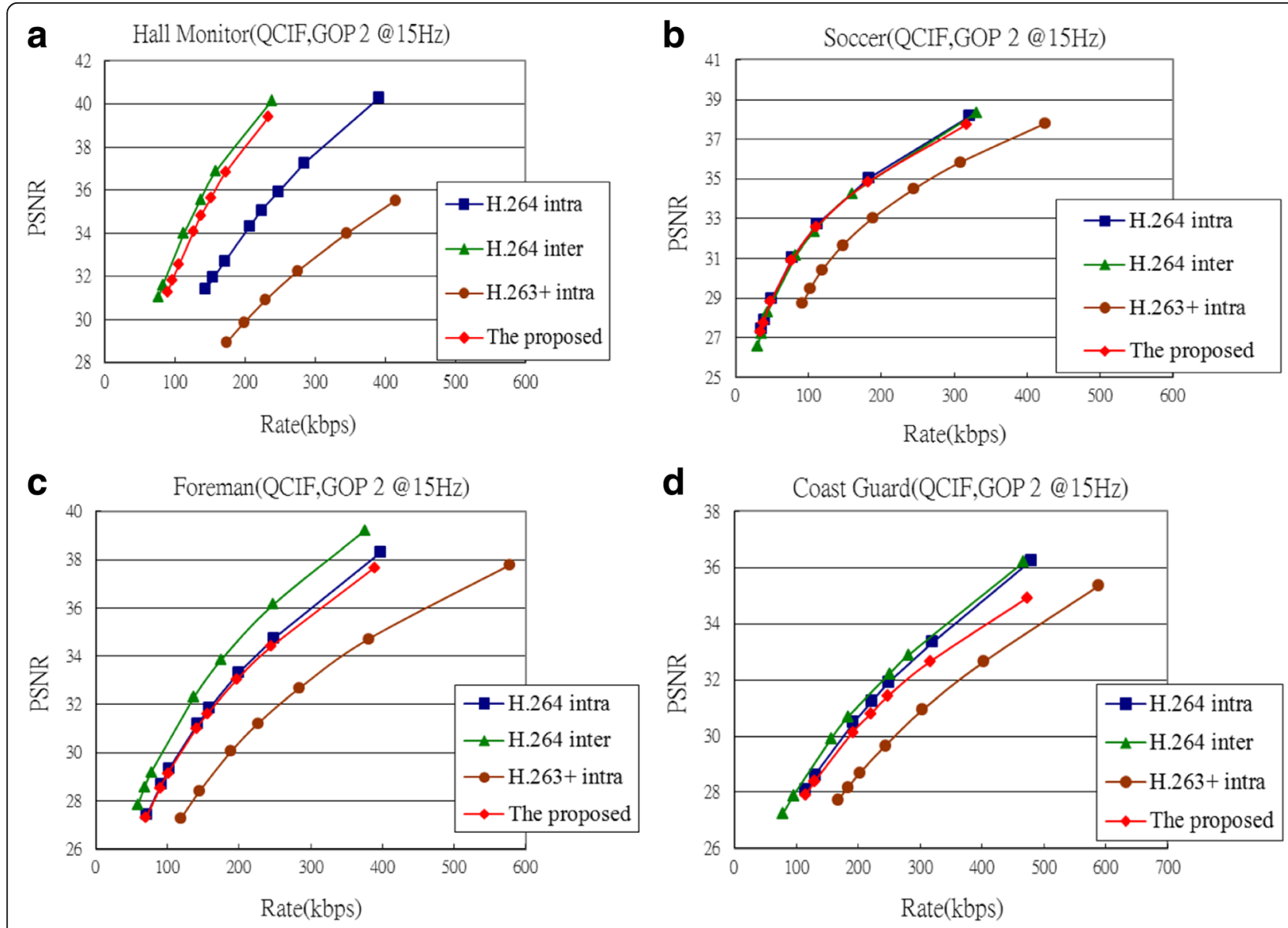

Fig. 10 The RD performance of the proposed scheme compared to that of H.264/AVC and H.263+ in GOP 2 
in GOP 2, the proposed scheme's (red line) performance is compared with that of DISCOVER in most video test sequences. Notably, Coast Guard and Soccer have been polarized. The proposed scheme's performance for Coast Guard is lower than that of DISCOVER and is $2 \mathrm{~dB}$. However, the proposed scheme's performance for Soccer is higher than that of DISCOVER at 3 to $4 \mathrm{~dB}$. The performance for Hall Monitor and Foreman is comparable with that of the DISCOVER codec. Conclusions regarding performance suggest that the proposed scheme is better than DISCOVER in GOP 8 but is comparable with DISCOVER in GOP 2, as seen in Fig. 9.

From this result, it is clear that the proposed scheme is more advantages, since in all types of video test sequences, even low motion or high motion video sequences, the proposed scheme's performance remains consistent at a certain level. Unlike traditional DVCs that only perform well under certain beneficial video test sequences, the proposed scheme can consistently exhibit improved performance. However, in some disadvantageous video test sequences, e.g., DISCOVER codec in Soccer, its performance will obviously become poor. In addition, the proposed scheme exhibits more stable performance and a small difference in different GOP length. These two advantages cannot be achieved with traditional DVCs.

In addition to comparison with the DISCOVER codec, the proposed scheme must also be compared with traditional video coding, e.g., H.264/AVC and H.263+ video coding standard. This comparison more clearly shows the superiority of the proposed scheme. Thus, H.264/AVC intra, H.264/AVC inter with no motion, and H.263+ are three standards chosen for comparison. Overall, the proposed scheme's performance is better than that of H.263+ and close to that of H.264/AVC, and from the curve, it can be seen that the proposed scheme's performance approaches the level of H.264/AVC inter with no motion.

In GOP 2, Hall monitor and Soccer, the proposed scheme's curve (red line) is closer to H.264/AVC inter; with traditional DVC contrast, most DVC solutions could not simultaneously result in such good performance between these two video test sequences. However, the proposed scheme's performance with Foreman and Coast Guard is closer to and slightly lower than H.264/AVC intra. Therefore, it is regarded as a fairly good performance.

In GOP 8, the performance is the same as in GOP 2 for the four video test sequences. Therefore, from the
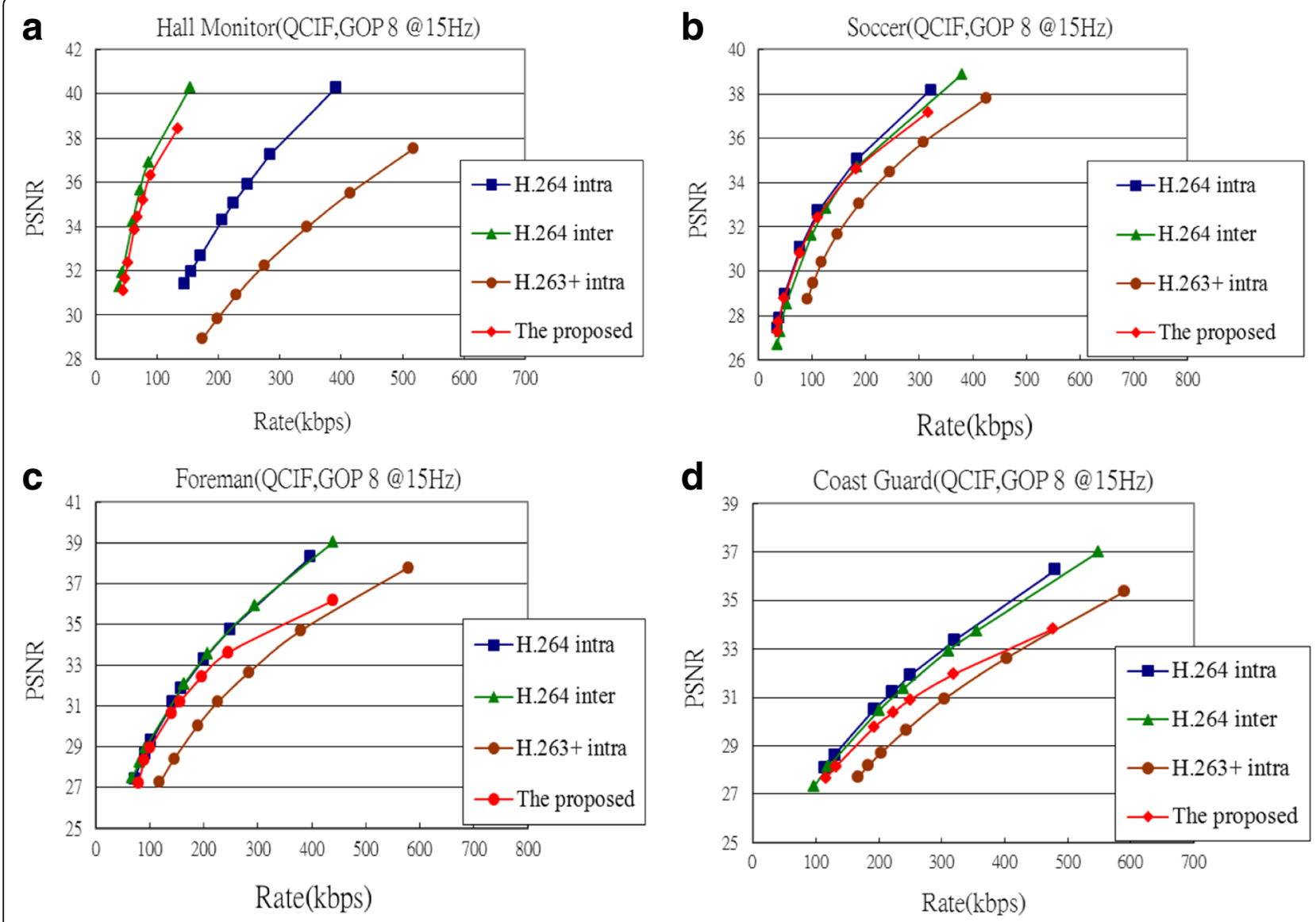

Fig. 11 The RD performance of the proposed scheme compared to that of H.264/AVC and H.263+ in GOP 8 
results of Figs. 10 and 11, it is clear that the DVCs' performance is lower than that of the traditional H.264/AVC video coding. This is because the usage of DVC encoder complexity is lower than the conventional H.264/AVC video coding by almost 10 to 20 times. Thus, it is very difficult to attain the same level of performance at this stage. Of course, this also clearly highlights the dilemma faced in current DVC development: because computational computation at the encoder is reduced too much, the performance is still between H.264/AVC intra and inter with no motion, and it is difficult to improve on this.

Furthermore, in order to prove that the proposed could still maintain the same situation in the H.265/ HEVC standard, same four video testing sequences are selected for comparison, and the PB-based LVC uses H.265/HEVC intra as its conventional intra frame encoder and decoder. In GOP length 2 and 8, the performance is the same with the H.264/AVC standard for the all video sequences. Therefore, from the results of Fig. 12, it is clear that the performance is slightly lower than that of the traditional H.265/HEVC intra except Hall Monitor, which is the same quality in the H.264/ AVC video sequences.

\subsection{Computational complexity}

The encoding computational complexity in the proposed scheme is higher (worse) than that of the DISCOVER codec, except with Hall Monitor in GOP 8 because the proposed design adopts partial inter frame encoding rather than only using the intra frame encoding of DISCOVER. However, the proposed encoding ratio differs slightly from that of DISCOVER in GOP 2 by 0.6 to 0.7 . Hall Monitor in GOP 8 is less than $1 / 2$ of the intra frame encoding because it uses a sub-framing function; thus, the computational complexity is lower, detail depicted in Table 1.

In the decoding, the proposed computational complexity is much lower (better) than that of DISCOVER, which uses a high complexity error correction decoding. As a result, the decoding time is one hundred to even three thousand times that of the H.264/AVC video decoding. The high-complexity error correction decoding accounts for over $90 \%$ of decoding time. Therefore, the DISCOVER codec causes difficulties in real time system design as there is a high level of complexity at the decoder. The proposed scheme performs well in this case, as its complexity increases by only $7 \sim 18 \%$ with the
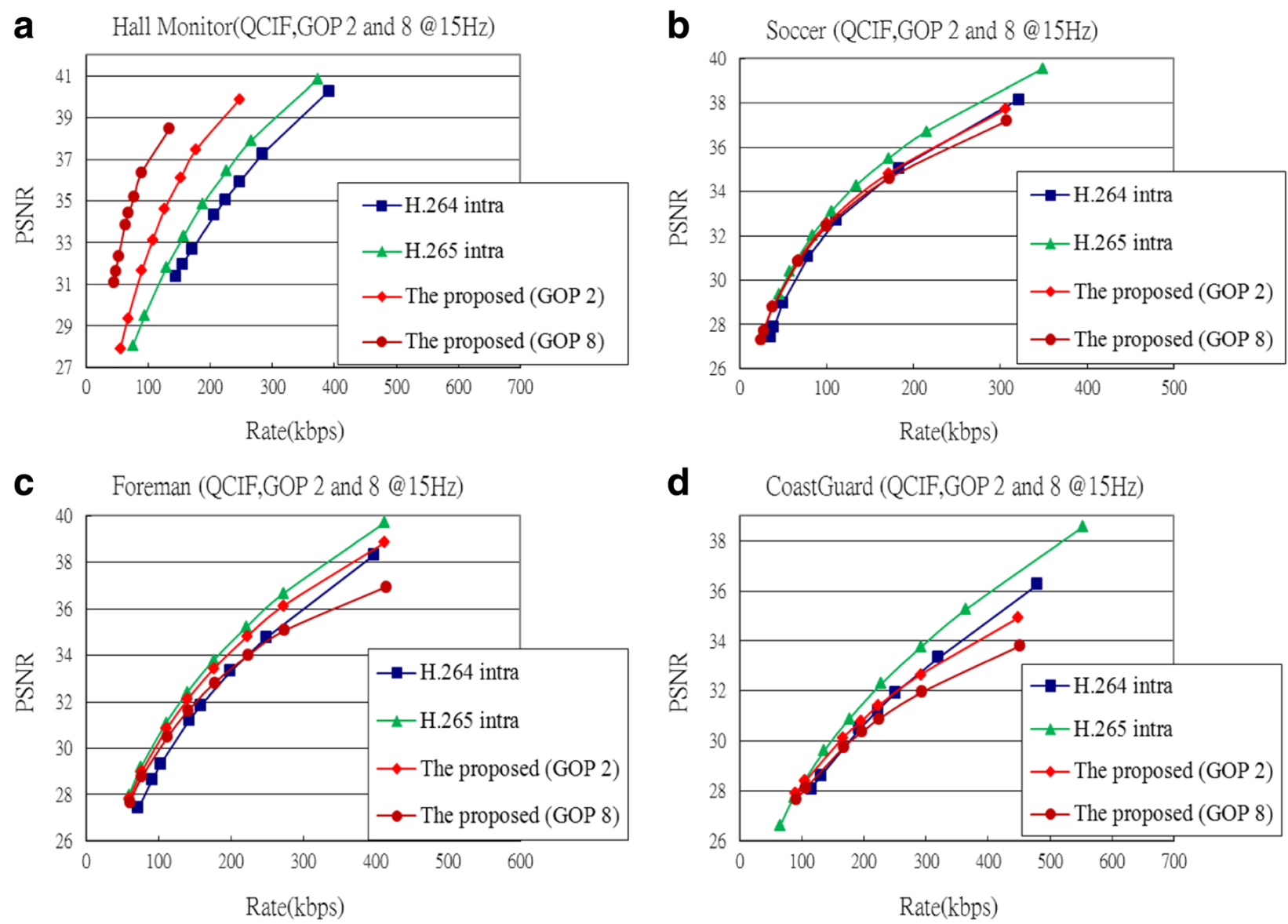

Fig. 12 The RD performance of the proposed scheme compared to that of H.265/HEVC 
Table 1 The encoder time ratio of the proposed PB-based LVC and DISCOVER codecs

\begin{tabular}{|c|c|c|c|c|}
\hline \multicolumn{5}{|c|}{ Encoding time complexity ratios (S) } \\
\hline \multirow[t]{2}{*}{$\overline{Q P}$} & $\mathrm{H} .264 \mathrm{i}$ & VER & H.264 i & \\
\hline & GOP 2 & GOP 8 & GOP 2 & GOP 8 \\
\hline
\end{tabular}

\section{Hall(QCIF@15Hz)}

$37 \quad 17$

$\begin{array}{ll}36 & 1.7\end{array}$

$36 \quad 1.7$

$33 \quad 17$

$33 \quad 1.71$

$31 \quad 1.71$

$29 \quad 1.7$

$24 \quad 1.73$

1.73

4.28

4.22

4.22

4.24

4.24

4.22

4.23

4.21

Soccer(QCIF@15Hz)

$\begin{array}{ll}44 & 1.66 \\ 43 & 1.67 \\ 41 & 1.66 \\ 36 & 1.68 \\ 36 & 1.68 \\ 34 & 1.69 \\ 31 & 1.69 \\ 25 & 1.73\end{array}$

Foreman(QCIF@15Hz)

$\begin{array}{ll}42 & 1.68 \\ 40 & 1.69 \\ 39 & 1.69 \\ 36 & 1.71 \\ 35 & 1.71 \\ 33 & 1.71 \\ 31 & 1.73 \\ 26 & 1.75\end{array}$

CoastGuard(QCIF@15Hz)

\begin{tabular}{lllll}
38 & 1.7 & 4.23 & 1 & 1 \\
37 & 1.71 & 4.23 & 1 & 1 \\
37 & 1.71 & 4.18 & 1 & 1 \\
34 & 1.72 & 4.33 & 1.01 & 1.01 \\
33 & 1.74 & 4.34 & 1 & 1.01 \\
31 & 1.75 & 4.41 & 1 & 1 \\
30 & 1.74 & 4.35 & 1 & 1.01 \\
26 & 1.76 & 4.45 & 1.01 & 1.01 \\
\hline
\end{tabular}

H.264/AVC video decoding. This is the main reason for using the proposed PBMA instead of the traditional DVC for error correction decoding. As such, the proposed application can enable a more efficient real-time processing environment, as shown in Table 2.
Table 2 The decoder time ratio of the proposed PB-based LVC and DISCOVER codecs

\begin{tabular}{|c|c|c|c|c|}
\hline \multicolumn{5}{|c|}{ Decoding time complexity ratios $(S)$} \\
\hline \multirow[t]{2}{*}{$\overline{\mathrm{QP}}$} & \multicolumn{2}{|c|}{ DISCOVER/H.264 intra } & \multicolumn{2}{|c|}{ Ours/H.264 intra } \\
\hline & $\overline{\mathrm{GOP}} 2$ & GOP 8 & GOP 2 & GOP 8 \\
\hline \multicolumn{5}{|c|}{ Hall(QCIF@15Hz) } \\
\hline 37 & 209 & 373 & 1.17 & 1.08 \\
\hline 36 & 223 & 395 & 1.17 & 1.1 \\
\hline 36 & 234 & 417 & 1.16 & 1.09 \\
\hline 33 & 306 & 660 & 1.17 & 1.09 \\
\hline 33 & 309 & 653 & 1.17 & 1.08 \\
\hline 31 & 397 & 876 & 1.16 & 1.08 \\
\hline 29 & 455 & 985 & 1.16 & 1.11 \\
\hline 24 & 581 & 1214 & 1.15 & 1.07 \\
\hline
\end{tabular}

Soccer(QCIF@15Hz)

$\begin{array}{lllll}44 & 745 & 1358 & 1.17 & 1.15 \\ 43 & 758 & 1399 & 1.18 & 1.16 \\ 41 & 888 & 1680 & 1.17 & 1.15 \\ 36 & 1303 & 2483 & 1.17 & 1.15 \\ 36 & 1399 & 2634 & 1.17 & 1.15 \\ 34 & 1690 & 3129 & 1.15 & 1.13 \\ 31 & 1810 & 3390 & 1.18 & 1.22 \\ 25 & 2117 & 4048 & 1.18 & 1.17\end{array}$

Foreman(QCIF@15Hz)

\begin{tabular}{lllll}
42 & 428 & 959 & 1.17 & 1.16 \\
40 & 470 & 1035 & 1.18 & 1.15 \\
39 & 536 & 1205 & 1.17 & 1.17 \\
36 & 830 & 2008 & 1.18 & 1.14 \\
35 & 928 & 2194 & 1.17 & 1.15 \\
33 & 1186 & 2666 & 1.16 & 1.16 \\
31 & 1285 & 3033 & 1.17 & 1.18 \\
26 & 1695 & 3979 & 1.16 & 1.16 \\
CoastGuard(QCIF@15Hz) & & & \\
38 & 277 & 636 & 1.18 & 1.17 \\
37 & 307 & 663 & 1.18 & 1.15 \\
37 & 329 & 761 & 1.18 & 1.15 \\
34 & 485 & 1322 & 1.17 & 1.16 \\
33 & 484 & 1370 & 1.18 & 1.17 \\
31 & 665 & 1856 & 1.18 & 1.16 \\
30 & 855 & 2203 & 1.17 & 1.16 \\
26 & 1282 & 3058 & 1.15 & 1.12 \\
\hline
\end{tabular}

\subsection{Computational occupation time}

Next, the functional block computational complexity time consumption needed further analysis and was calculated in percentage. The encoder distinguished three parts from Fig. 6, the first is the functional block 
classifier and skip block mask, the second is the functional block rearrangement and sub-framing, and the third is the H.264/AVC intra frame encoding and similarly for comparison in GOP 2 and 8. Overall, because the DVC design aim is low-complexity encoding, the highest computational complexity occurs with the
H.264/AVC intra encoding, and execution time accounts for more than $99.5 \%$. Although the proposed scheme adopts partial inter frame encoding, it exhibits higher complexity than the traditional DVC intra frame encoding. However, it is observed that the increasing partial inter frame coding time is lower than $0.5 \%$, from Table 3 ,

Table 3 The encoder time percentage of the proposed PB-based LVC

\begin{tabular}{|c|c|c|c|c|c|c|}
\hline \multicolumn{7}{|c|}{ Percentage of the encoding time } \\
\hline \multirow[b]{2}{*}{ QP } & \multicolumn{3}{|c|}{ GOP 2} & \multicolumn{3}{|c|}{ GOP 8} \\
\hline & $\begin{array}{l}\text { Classifier and skip } \\
\text { block mask }\end{array}$ & $\begin{array}{l}\text { Rearrangement and } \\
\text { sub-framing }\end{array}$ & H.264/AVC intra & $\begin{array}{l}\text { Classifier and skip } \\
\text { block mask }\end{array}$ & $\begin{array}{l}\text { Rearrangement and } \\
\text { sub-framing }\end{array}$ & $\begin{array}{l}\text { H.264/AVC } \\
\text { intra }\end{array}$ \\
\hline \multicolumn{7}{|c|}{ Hall (\%, QCIF@15Hz) } \\
\hline 37 & 0.36 & & 99.64 & 0.55 & 0.22 & 99.08 \\
\hline 36 & 0.36 & & 99.64 & 0.55 & 0.22 & 99.09 \\
\hline 36 & 0.35 & & 99.65 & 0.54 & 0.22 & 99.09 \\
\hline 33 & 0.34 & & 99.66 & 0.53 & 0.21 & 99.12 \\
\hline 33 & 0.33 & & 99.67 & 0.53 & 0.21 & 99.12 \\
\hline 31 & 0.33 & & 99.67 & 0.52 & 0.21 & 99.14 \\
\hline 29 & 0.32 & & 99.68 & 0.51 & 0.2 & 99.16 \\
\hline 24 & 0.29 & & 99.71 & 0.47 & 0.19 & 99.21 \\
\hline \multicolumn{7}{|c|}{ Soccer (\%, QCIF@15Hz) } \\
\hline 44 & 0.39 & & 99.61 & 0.46 & & 99.54 \\
\hline 43 & 0.39 & & 99.61 & 0.46 & & 99.54 \\
\hline 41 & 0.38 & & 99.62 & 0.45 & & 99.55 \\
\hline 36 & 0.36 & & 99.64 & 0.42 & & 99.58 \\
\hline 36 & 0.36 & & 99.64 & 0.42 & & 99.58 \\
\hline 34 & 0.34 & & 99.66 & 0.4 & & 99.6 \\
\hline 31 & 0.31 & & 99.69 & 0.36 & & 99.64 \\
\hline 25 & 0.26 & & 99.74 & 0.3 & & 99.7 \\
\hline \multicolumn{7}{|c|}{ Foreman (\%, QCIF@15Hz) } \\
\hline 42 & 0.34 & & 99.66 & 0.43 & & 99.57 \\
\hline 40 & 0.33 & & 99.67 & 0.41 & & 99.59 \\
\hline 39 & 0.32 & & 99.68 & 0.41 & & 9959 \\
\hline 36 & 0.3 & & 99.7 & 0.38 & & 99.62 \\
\hline 35 & 0.29 & & 99.71 & 0.37 & & 99.63 \\
\hline 33 & 0.28 & & 99.72 & 0.35 & & 99.65 \\
\hline 31 & 0.26 & & 99.74 & 0.33 & & 99.67 \\
\hline 26 & 0.22 & & 99.78 & 0.27 & & 99.73 \\
\hline \multicolumn{7}{|c|}{ Coast Guard (\%, QCIF@15Hz) } \\
\hline 38 & 0.37 & & 99.63 & 0.42 & & 99.58 \\
\hline 37 & 0.36 & & 99.64 & 0.41 & & 99.59 \\
\hline 37 & 0.36 & & 99.64 & 0.41 & & 99.59 \\
\hline 34 & 0.33 & & 99.67 & 0.38 & & 99.62 \\
\hline 33 & 0.32 & & 99.68 & 0.36 & & 99.64 \\
\hline 31 & 0.31 & & 99.69 & 0.35 & & 99.65 \\
\hline 30 & 0.28 & & 99.72 & 0.32 & & 99.68 \\
\hline 26 & 0.24 & & 99.76 & 0.28 & & 99.72 \\
\hline
\end{tabular}


and below one in 200. Therefore, the time proportion is extremely low.

Hall monitor in GOP 8, however, is a low motion video sequence, and most blocks are skipped; thus, functional block sub-framing is used. The encoder is therefore partially occupied with the sub-framing block during processing time.
In addition, the decoding time occupation analysis is divided into four parts from Fig. 7. First is functional block H.264/AVC intra frame decoding, second is functional block sub-frame recovery and inverse rearrangement, third is functional block block padding, and the final is functional block pixel padding. Overall, the

Table 4 The decoder time percentage of the proposed PB-based LVC

\begin{tabular}{|c|c|c|c|c|c|c|c|c|}
\hline \multicolumn{9}{|c|}{ Percentage of the decoding time } \\
\hline \multirow[b]{2}{*}{ QP } & \multicolumn{4}{|c|}{ GOP 2} & \multicolumn{4}{|c|}{ GOP 8} \\
\hline & $\begin{array}{l}\text { H.264/AVC } \\
\text { intra }\end{array}$ & $\begin{array}{l}\text { Sub-frame recovery and } \\
\text { inverse rearrange }\end{array}$ & $\begin{array}{l}\text { Block } \\
\text { padding }\end{array}$ & $\begin{array}{l}\text { Pixel } \\
\text { padding }\end{array}$ & $\begin{array}{l}\text { H.264/AVC } \\
\text { intra }\end{array}$ & $\begin{array}{l}\text { Sub-frame recovery and } \\
\text { inverse rearrange }\end{array}$ & $\begin{array}{l}\text { Block } \\
\text { padding }\end{array}$ & $\begin{array}{l}\text { Pixel } \\
\text { padding }\end{array}$ \\
\hline \multicolumn{9}{|c|}{ Hall (\%, QCIF@15Hz) } \\
\hline 37 & 84.3 & & 9.42 & 6.28 & 96.34 & 0.73 & 1.83 & 1.1 \\
\hline 36 & 84.3 & & 9.42 & 6.28 & 96.3 & 0.74 & 1.85 & 1.11 \\
\hline 36 & 84.31 & & 9.41 & 6.27 & 96.3 & 0.74 & 1.85 & 1.11 \\
\hline 33 & 84.5 & & 9.3 & 6.2 & 96.39 & 0.72 & 1.81 & 1.08 \\
\hline 33 & 84.26 & & 9.44 & 6.3 & 96.36 & 0.73 & 1.82 & 1.09 \\
\hline 31 & 84.34 & & 9.4 & 6.27 & 96.27 & 0.75 & 1.86 & 1.12 \\
\hline 29 & 84.32 & & 9.41 & 6.27 & 96.4 & 0.72 & 1.8 & 1.08 \\
\hline 24 & 84.38 & & 9.37 & 6.25 & 96.4 & 0.72 & 1.8 & 1.08 \\
\hline \multicolumn{9}{|c|}{ Soccer (\%, QCIF@15Hz) } \\
\hline 44 & 84.51 & & 9.3 & 6.2 & 86.02 & & 8.39 & 5.59 \\
\hline 43 & 84.55 & & 9.27 & 6.18 & 85.41 & & 8.76 & 5.84 \\
\hline 41 & 84.37 & & 9.38 & 6.25 & 85.82 & & 8.51 & 5.67 \\
\hline 36 & 84.72 & & 9.17 & 6.11 & 85.61 & & 8.63 & 5.76 \\
\hline 36 & 84.72 & & 9.17 & 6.11 & 85.61 & & 8.63 & 5.76 \\
\hline 34 & 84.59 & & 9.24 & 6.16 & 85.82 & & 8.51 & 5.67 \\
\hline 31 & 84.73 & & 9.16 & 6.11 & 86.46 & & 8.12 & 5.42 \\
\hline 25 & 84.88 & & 9.07 & 6.05 & 85.96 & & 8.42 & 5.62 \\
\hline \multicolumn{9}{|c|}{ Foreman (\%, QCIF@15Hz) } \\
\hline 42 & 84.73 & & 9.16 & 6.11 & 85.89 & & 8.47 & 5.64 \\
\hline 40 & 84.88 & & 9.07 & 6.05 & 85.84 & & 8.5 & 5.66 \\
\hline 39 & 84.75 & & 9.15 & 6.1 & 85.78 & & 8.53 & 5.69 \\
\hline 36 & 84.65 & & 9.21 & 6.14 & 85.77 & & 8.54 & 5.69 \\
\hline 35 & 84.7 & & 9.18 & 6.12 & 86.09 & & 8.35 & 5.56 \\
\hline 33 & 84.81 & & 9.11 & 6.08 & 86.06 & & 8.36 & 5.58 \\
\hline 31 & 84.87 & & 9.08 & 6.05 & 86.31 & & 8.22 & 5.48 \\
\hline 26 & 84.98 & & 9.01 & 6.01 & 86.2 & & 8.28 & 5.52 \\
\hline \multicolumn{9}{|c|}{ Coast Guard (\%, QCIF@15Hz) } \\
\hline 38 & 84.6 & & 9.24 & 6.16 & 85.71 & & 8.57 & 5.71 \\
\hline 37 & 84.37 & & 9.38 & 6.25 & 85.72 & & 8.57 & 5.71 \\
\hline 37 & 84.37 & & 9.38 & 6.25 & 85.72 & & 8.57 & 5.71 \\
\hline 34 & 84.37 & & 9.38 & 6.25 & 85.6 & & 8.64 & 5.76 \\
\hline 33 & 84.51 & & 9.29 & 6.2 & 85.79 & & 8.53 & 5.68 \\
\hline 31 & 84.63 & & 9.22 & 6.15 & 85.75 & & 8.55 & 5.7 \\
\hline 30 & 84.72 & & 9.17 & 6.11 & 85.85 & & 8.49 & 5.66 \\
\hline 26 & 84.84 & & 9.09 & 6.06 & 85.94 & & 8.44 & 5.63 \\
\hline
\end{tabular}


decoder exhibits the same performance as the encoder, in that the greatest complexity occurs with H.264/AVC intra decoding, accounting for about $85 \%$, while the second highest execution time occurs with functional block padding and occupies about $8 \sim 9 \%$, from Table 4 . The decoder's improvement performance relies on this function, and the two important algorithms, PBMA and mode decision at decoder, also perform in this part.

\section{Conclusions}

In this paper, a PB-based LVC architecture was proposed, which compensates for the current highcomplexity decoder and lower performance DVC schemes. Following the experiment results, it is clear that the proposed architecture is able to greatly reduce the decoder complexity by a hundred-fold in addition to other marginal performance improvements. Moreover, the PBMA and four flexible block type mode decision scheme is able to effectively transfer the motion estimation and the mode decision algorithms to the decoder. Its performance can approach that of H.264/AVC video coding, and is better than traditional DVC solutions.

\section{Funding}

This work has been funded by grants 5132-28Y, from the Education Ministry of Taiwan R.O.C Government.

\section{Authors' contributions}

$T L$ and FsT conceived and designed the research. TL performed the experiments and analyzed the data. TL and FsT wrote and edited the manuscript. Both authors read and approved the final manuscript.

\section{Competing interests}

The authors declare that they have no competing interests.

\section{Publisher's Note}

Springer Nature remains neutral with regard to jurisdictional claims in published maps and institutional affiliations.

\section{Author details}

'The Electrical Engineering Department of National Sun Yat-sen University, 70 Lienhai Rd., Kaohsiung 80424, Taiwan, Republic of China. ${ }^{2}$ The Institute of Communication Engineering of National Sun Yat-sen University, 70 Lienhai Rd, Kaohsiung 80424, Taiwan, Republic of China.

Received: 10 May 2015 Accepted: 18 April 2017

Published online: 22 May 2017

\section{References}

1. J Slepian, J Wolf, Noiseless coding of correlated information sources. IEEE Trans. Inf. Theory. 19 (4), 471-480 (1973)

2. A Wyner, J Ziv, The rate-distortion function for source coding with side information at the decoder. IEEE Trans. Inf. Theory. 22 (1), 1-10 (1976)

3. B Girod, A Aaron, S Rane, D Rebollo-Monedero, Distributed video coding. Proc. IEEE. 93 (1), 71-83 (2005)

4. X Artigas, J Ascenso, M Dalai, S Klomp, D Kubasov, M Ouaret, The DISCOVER Codec: Architecture, Techniques and Evaluation. (Picture Coding Symposium, Lisbon, 2007)

5. C Brites, J Ascenso, J Pedro, F Pereira, Evaluating a feedback channel based transform domain Wyner-Ziv video codec. Signal Process. Image Commun. 23, 269-297 (2008)

6. R Puri, A Majumdar, K Ramchandran, PRISM: a video coding paradigm with motion estimation at the decoder. IEEE Trans. Image Process. 16(10), 2436$2448(2007)$
7. CC Chiu, SY Chien, CH Lee, VS Somayazulu, YK Chen, Hybrid distributed video coding with frame level coding mode selection. 19th IEEE International Conference on Image Processing, 1561-1564 (2012)

8. CC Chiu, HF Wu, SY Chien, CH Lee, VS Somayazulu, YK Chen, Hardware Architecture Design of Hybrid Distributed Video Coding with Frame Level Coding Mode Selection. Asia-Pacific Signal \& Information Processing Association Annual Summit and Conference, 1-4 (2012)

9. J Ascenso, C Brites, F Pereira, Improving frame interpolation with spatial motion smoothing for pixel domain distributed video coding. 5th EURASIP Conference on Speech and Image Processing, Multimedia Communications and Services. (Slovak Republic, Smolenice, 2005), 1-6

10. T Clercks, A Munteanu, J Cornelis, P Schelkens, Distributed Video Coding with Shared Encoder/Decoder Complexity. IEEE International Conference on Image Processing, San Antonio, TX. 6, VI-417-VI-420 (2007)

11. H Chen, E Steinbach, Flexible Distribution of Computational Complexity Between the Encoder and the Decoder in Distributed Video Coding. (IEEE Int. Conf. Multimedia and Expo, Hannover, Germany, 2008),pp. 801-804

12. F Dufaux, T Ebrahimi, Encoder and Decoder Side Global and Local Motion Estimation for Distributed Video Coding. 2010 IEEE International Workshop on Multimedia Signal Processing, 339-344 (2010)

13. A Abou-Elailah, F Dufaux, J Farah, M Cagnazzo and B Pesquet-Popescu, Fusion of Global and Local Motion Estimation for Distributed Video Coding, IEEE Trans. Circuits Syst. Video Technol. 23 (1) (2013)

14. DG Lowe, Distinctive image features from scale-invariant keypoints. Int. J. Comput. Vision 60(2), 91-110 (2004)

15. Y-J Wang, S-L Hsu, T-Y Cheng, C-H Lee, S-Y Chien, Feedback-Channel-Free Distributed Video Coding with Dynamic Skip-Mode Threshold. International Conference on Image Processing, (2012)

16. Y-J Wang, S-L Hsu, T-Y Cheng, C-H Lee, S-Y Chien, Low-Complexity Feedback-Channel-Free Distributed Video Coding with Enhanced Classifier. IEEE International Symposium on Circuits and Systems. (2013)

17. EG lain, H Richardson, 264 and MPEG-4 Video Compression: Video Coding for Next-generation Multimedia. (John Wiley \& Sons, 2003)

18. T Wiegand, GJ Sullivan, G Bjontegaard, A Luthra, Overview of the H.264/AVC video coding standard. IEEE Trans. Circuits Syst. Video Technol. 13(7), 560576 (2003)

19. J Zhang, JF Arnold MR Frater, A cell-loss concealment technique for MPEG-2 coded video. IEEE Trans. Circuits Syst. Video Technol. 10(4), 659-665 (2000)

20. AA Efros, TK Leung, Texture Synthesis by Nonparametric Sampling. IEEE Int. Conf. Computer Vision., 1033-1038 (1999)

21. S Rane, G Sapiro, M Bertalmio, Structure and texture filling-in of missing image blocks in wireless transmission and compression applications. IEEE Trans. Image Process. 296-303 (2003)

\section{Submit your manuscript to a SpringerOpen ${ }^{\mathcal{O}}$ journal and benefit from:}

- Convenient online submission

- Rigorous peer review

- Immediate publication on acceptance

- Open access: articles freely available online

- High visibility within the field

- Retaining the copyright to your article

Submit your next manuscript at springeropen.com 